\title{
Recording of tactile observing responses for the study of selective attention'
}

\author{
S. RYDBERG, R. KASHDAN AND T, TRABASSO 2 \\ UNIVERSITY OF CALIFORNIA, LOS ANGELES
}

Apparatus is described, complete with circuit diagram, which provides continuous measurement of selective attention to spatially separate, tactile stimuli. Tactile and other responses to physical objects are recorded electrically. This permits direct observation of each observing response throughout the duration of a trial. An ink-on-paper record yields types, durations, frequencies, patterns, latencies and correctness of responses. This device has potential usage in both learning and psychophysical experinentation, particularly on the direction and/or degree of attention. Some preliminary data in a discrimination learning task are given which show that Ss spend less time touching irrelevant stimuli near terminal learning.

In this paper we shall describe a device which permits direct, objective and continuous measurement of selective attention. Specifically, we are concerned with attention as it is manifested in overt tactile observing responses. These responses can be restricted or not, active (haptic; Gibson, 1962) or passive. The device produces an automatic ink-on-paper record of any touch events under study. Touch pressure does not have to be critical, since the electric circuitry of the device can be adjusted to detect the slightest touch. The paper record shows whether, when, and for how long a stimulus object, or part thereof, is touched. In addition, one can simultaneously record choice responses, reinforcing operations and other experimental events. With such objective recording of observing responses, tactile experiments could be preliminary, complementary, and sometimes superior to any analogous experiments based on visual and other stimuli. Many tactile stimuli seem to be more novel and less associated with verbal labels in unprepared human adults.

The apparatus may be used in a number of psychophysical and learning situations. In particular, it would be useful when the direction and/or degree of attention are primary factors of interest.

\section{Method}

Recording of the S's tactile activity is achieved by means of electrically conductive stimulus objects mounted and spatially separated, e.g., as in Fig. 1, on a nonconductive surface. The $\mathrm{S}$ can be restricted to touching only one object at a time. One way to do this is to cover his hand by an ordinary sock with a hole allowing only one finger to protrude. When an object is in place on the stimulus presentation board, it connects to the input of a transistor amplifier circuit. A circuit diagram (Fig. 2) and a circuit description will be given later. By means of an armband electrode, $\mathrm{S}$ is connected to another part of the circuit. When $S$ touches the object, an unnoticeable current flows between $\mathrm{S}$ and the object. This current is amplified sufficiently to operate a relay closing a circuit that deflects a pen on an event recorder such as Gerbrands or Esterline Angus. This deflection indicates that the corresponding object is being touched. When $S$ stops touching the object, the circuits are broken and the recorder pen returns to its baseline.

Figure 1 shows schematically how we have used this device in a discrimination learning experiment on selective attention, although the method is more generally applicable. In the figure the $S$ has felt stimulus objects, which are raised off the surface of a wooden triangular block (Fig. 1a). There is one stimulus object in each corner. Each object represents one of three possible values of each of three stimulus dimensions: size of a circle, angle between two matchsticks, and texture of a triangle; this gives $3^{3}=27$ differentblocks. The blocks are presented to $S$ in a random order. For a given $\mathrm{S}$, dimension positions are fixed. His task is to find out that a dimension(s) is relevant and which of

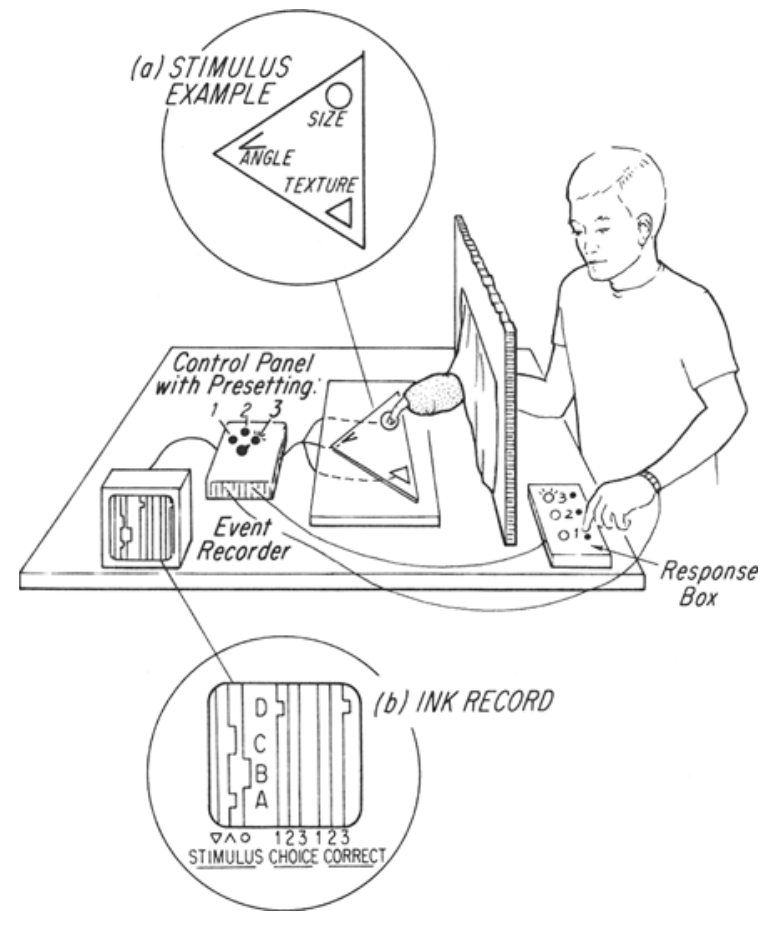

Fig. 1. Schematic example of touch recording setup, with enlarged (a) stimulus example and (b) ink record of touching, choice response, and reinforcement. 
three response buttons he should press for a given value of the relevant dimension(s). When $\mathrm{S}$ presses a button, he completes a circuit, which has been preset by $E$ on a control panel, which lights up a lamp next to the correct button. Figure $1 b$ shows that $S$ has first touched (A) the angle briefly, then (B) the circle, then (C) the angle again, then (D) pressed button 1 and the feedback lamp informed him that button 3 was correct.

The moment-by-moment monitoring achieved by the device makes possible moment-by-moment manipulation of observing behavior. One possibility is to change the circuitry so as to include automatic or experimenter-administered reinforcement contingent upon observing and/or other responses. If programming units are added, certain perceptual scanning patterns can be reinforced automatically.

Another possibility is to supplement or replace the ink recording by magnetic tape recording structured to be compatible with a computer data analysis program.

In our case the paper record has provided the following kinds of data for each trial:

(1) which stimulus objects were touched (observing responses)

(2) when each observing response occurred

(3) how often an observing response occurred

(4) sequences or patterns of observing responses

(5) durations of observing responses

(6) occurrences, frequencies, patterns, and durations of non-touching

(7) various latencies

(8) choice responses (button-pressing); reinforcements, and their durations

(9) trial durations

\section{Results}

Although we would like to present individual and group data on each of these variables, such reporting has to await future publication on our part. However, to illustrate that changes of observing behavior do occur during learning, data from one of our preliminary experiments, with $18 \mathrm{Ss}$, will be given which indicate that Ss tend to touch the irrelevant stimulus less towards the end of a discrimination learning task. Only one stimulus dimension out of the three on a block was irrelevant. We divided the pre-criterion learning period into four quarters. Touching time on the irrelevant stimulus dimension, calculated as a percentage of the total time spent touching the stimulus objects in a trial, average $32,31,32$, and 26 per quarter, compared to 18 during the criterion run (10 consecutive correct choice responses). Touching of the irrelevant stimulus was at chance during the first three quarters, and fell off during the fourth quarter and the criterion run.

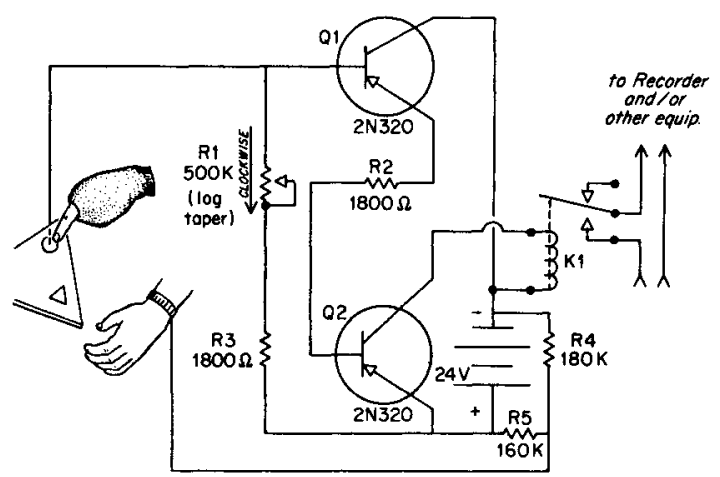

Fig. 2. Touch detector amplifier circuitry for one stimulus. ( $K 1$ is Lafayette No. $99 R 6093$. All resistors are rated at 0.5 watts.)

\section{Detector circuitry}

Reinforcement circuitry and certain arrangements for automatic starting and stopping of the recorder are generally known and are not described.

Figure 2 shows the transistor amplifier circuit for one stimulus object. The nonconductive objects were covered with GC Electronics conductive paint, Silver Print No. 21-1. The painted surfaces connected to the underside of the block. When the block was in place, each corner made contact with its amplifier circuit. R1 adjusts the sensitivity of the circuit to touch. For normal operation, R1 should be turned clockwise until the slightest touch of the stimulus object closes the relay to activate the event recorder. If $R 1$ is turned too far clockwise, the relay will remain closed after the end of a touch. If desired, R1 can be turned counterclockwise so that only firm touches will be detected. Room temperature should be kept reasonably constant, otherwise $\mathrm{R} 1$ may have to be readjusted. The voltage between $S$ and the stimulus object is limited to about $10 \mathrm{v}$ so as to make the current through $\mathrm{S}$ unnoticeable.

\section{Reference}

Gibson, J. J. Observations on active touch. Psychol. Rev., 1962, $69,477-490$.

\section{Notes}

1. This research was supported by NSF grant No. 145 by the Committee on Research, University of California, Los Angeles, to $S$. Rydberg and $\mathrm{T}$. Trabasso and also by grant 5 R01-MH-08741-03 from the National Institutes of Health, USPHS, to T. Trabasso. 2. Any communication to the senior author should be directed to University of Stockholm, Box 23052, Stockholm 23, Stveden. The circuitry was designed by R. Kashdan. 